\title{
Performance of Opportunistic Routing in Low Duty-Cycle Wireless Sensor Networks
}

\author{
Nadjib Aitsaadi*, Bartłomiej Błaszczyszyn ${ }^{\S}$ and Paul Muhlethaler $\ddagger$ \\ ${ }^{*}$ LISSI, University of Paris-Est Creteil Val de Marne (UPEC): 122 rue Paul Armangot, 94400 Vitry sur Seine - France \\ $\S$ INRIA/ENS Paris: 23 Avenue d'Italie, 75214 PARIS CEDEX 13 - France \\ $\ddagger$ INRIA Paris Rocquencourt: Domaine de Voluceau - Rocquencourt - B.P. 105, 78153 Le Chesnay Cedex - France \\ nadjib.aitsaadi@u-pec.fr, bartek.blaszczyszyn@ens.fr, paul.muhlethaler@inria.fr
}

\begin{abstract}
In this paper, we propose three variants of WSN routing protocol: i) basic-opportunistic, ii) opportunistic with delay and iii) opportunistic with backtracking. The main idea is that a node transmits its packet to the next awake node as long as this decreases the remaining distance to the sink. The two last variants allow a packet to be discarded or moved further away from the sink if necessary. The performance evaluation is carried out by simulations and with analytical model based on a Poisson rain model. The comparison is based i) probability of packet delivery, ii) end-toend packet delay and iii) number of hops between the source and the sink node.
\end{abstract}

Keywords: WSN opportunistic routing, performance evaluation.

I. INTRODUCTION

Opportunistic routing has recently emerged and demonstrated both simplicity and scalability. It is worth noting that a node does not use a routing table. This kind of routing exploits the broadcast nature of wireless communication: when a node transmits a packet, all its active neighbours can receive it. However, only one neighbour should forward the packet. There are various means to build such a mechanism. It may be a self election process or the transmitter can select the next hop according to information about the nodes in its neighbourhood, see [1].

In this paper, we study the performance of opportunistic routing and how can be used in WSNs operating in a low duty-cycle mode. In contrast to previous work, "opportunism" is used to take advantage of awake nodes and not to benefit from all the receptions in the neighbourhood of a node such as in [2]. To maximise the network's lifetime, whenever possible nodes' transceivers are periodically and asynchronously switched off; the WSN operates with a low duty-cycle. However, we have to ensure that the network remains connected. This property can be obtained by calibrating the communication range or density of deployed sensors. We also have to guarantee that the packet delivery delay is acceptable, which leads to a constraint on the length of time that the sensors are turned off.

The main contributions of this paper are as follows. First, we define in detail the opportunistic cross-layer scheme that we use in the low duty-cycle WSN. This scheme is a combination of RI-MAC [3] and geographic opportunistic routing. In fact, we consider three opportunistic variants: i) basic-opportunistic, ii) opportunistic with delay, and iii) opportunistic with backtracking. When the WSN operates in a low duty-cycle mode, we compare the performance of the Dijkstra shortest path protocol with our opportunistic routing technique. Depending on the transmission range, we evaluate the i) packet delivery probability, ii) end-toend delivery time, iii) delay at each hop, and iv) and number of hops on the path to the sink. We compare the simulation results with those obtained by the proposed analytical model based on the Poisson rain model and we note a very good matching.
The remainder of this paper is organised as follows. The next describes the geographic opportunistic cross-layer scheme. In Section III, we introduce the network and analytical models for our proposal. In Section IV, we gauge the performance of the proposal analytically and by extensive simulations. Finally, Section $\mathrm{V}$ concludes the paper.

\section{OPPORTUNISTIC CROSS-LAYER SCHEMES IN LOW DUTY-CYCLE WSNS}

To save energy, sensor nodes are asynchronously turned off in the network. A sensor node's transceiver is active for one time unit and it sleeps for a given time period which is preferably large compared to the one unit of activity. We assume that the beginning of an active period is indicated by a beacon.

We use a technique where the transmitter waits for the receiver to wake up before sending the packet to it. This is the ReceiverInitiated MAC [3] protocol. In this approach, when a node awakes and switches on the transceiver, it sends a beacon to let its neighbours know that its transceiver is on. In order to avoid collisions, the awake node indicates in its beacon the collision window that must be used for the nodes which want to communicate with it. Thus, the sender uses CSMA and will not transmit if it senses a prior transmission.

RI-MAC must be complemented at the routing level and we propose to make use of a geographic opportunistic protocol. In that sense our approach is a cross-layer scheme. When a sensor wants to transmit a packet, it waits for the next awake sensor node and checks whether or not it reduces the remaining distance to the sink. If so, the packet is sent to this node just at the beginning of its active period. We consider three variants of the geographic opportunistic routing protocol, which conveys a packet as follows. First, each sensor node determines its geographic position. We assume that sensors are equipped with a GPS or apply geolocalization methods proposed in the literature [4]. Then, each node communicates its position to its neighbours and the sink node floods its coordinates in the whole network. This location can also be written in the sensor nodes when the network is set up. When a sensor node switches on its transceiver, it informs the neighbours by sending a beacon. To forward a packet from a node $\mathcal{N}_{i}$ to the sink $\mathcal{S}$, the next hop is selected according to the variant of opportunistic routing as explained below.

1) Basic-opportunistic: The next hop $\mathcal{N}_{i}$ is selected as the next active neighbour that decreases the remaining distance to the sink $\mathcal{S}$. If the remaining distance between the sink and the next awake node is not smaller than the distance between the sink and the current sensor node, the packet waits in $\mathcal{N}_{i}$ for possibly an unlimited period. This variant 
is greedy, the distance between the sink and the next sensor relay never increases.

2) Opportunistic with delay: This is similar to the basicopportunistic variant except that in each hop a packet can wait for, at most, a predefined and fixed duration. If a packet is not transmitted during this period, it will be discarded. In other words, a packet only waits for a limited amount of time in each node.

3) Opportunistic with backtracking: This is similar to the opportunistic with delay variant except that it is not greedy. If a next hop minimising the remaining distance is not found during the maximum waiting time, the packet is not discarded and the protocol allows the packet to initially move further away from the sink until a path to the destination is found. Moreover, a node $\mathcal{N}_{i}$ will be tagged as a forbidden hop in the future for the packet concerned (i.e. a packet cannot visit a node $\mathcal{N}_{i}$ again). We know that, in random networks, packets using greedy opportunistic routing can be blocked by holes. The opportunistic with backtracking protocol is a response to this issue.

When a sensor node has a packet to forward it does not turn off its transceiver until it has successfully transmitted the packet to the next hop towards the destination. These protocols require only a very limited computation power and memory.

\section{NETWORK AND ANALYTICAL MODELS}

We consider a target deployment area denoted by $\mathcal{A}$. We assume that $\mathcal{A}$ is a square unit area ${ }^{1}$. Sensor nodes are deployed in $\mathcal{A}$ and their positions are the points of a homogenous Poisson Point Process with density $\lambda$. We assume the same communication range throughout the network, denoted by $\mathcal{R}_{\text {com }}$. We consider a WSN with an asynchronously low duty-cycle. A sensor node's transceiver is active for one time unit and it sleeps for a time exponentially distributed with density $\lambda_{o f f}$.

We assume an infrequent event is being monitored. Only one packet can be transmitted within any sensor's neighbourhood. Hence, we can ignore collisions between packets. In order to generate a long path, we assume that a packet is sent from a sensor $\mathcal{O}$ located at $(0.1,0.1)$ to the sink node $\mathcal{S}$ deployed at $(0.9,0.9)$ (i.e. diagonal).

In what follows, we study the following parameters:

- probability of packet delivery, denoted by $P_{\text {path }}$,

- average end-to-end packet delay, denoted by $T_{t o t}$,

- average number of hops per path, denoted by $\mathcal{N}_{\text {hop }}$.

A direct analysis of the above network model does not seem to be possible. Therefore, we use a Poisson rain model [5] and we analyse the opportunistic with delay variant. To simplify, we assume that the maximum delay at each hop is equal to $1 / \lambda_{\text {off } f}$. The main difference with respect to the network model described above is that the nodes $\left\{N_{i}\right\}$ are not fixed in time. Instead, we may think of these nodes as being "born" at some time $T_{i}$ and being active one unit of time and "disappearing" immediately after. The joint space-time distribution of node locations and transmission instances $\Psi=\left\{\left(X_{i}, T_{i}\right)\right\}$ is modelled by a homogeneous Poisson point process in $2+1$ dimensions with intensity $\lambda_{s}=\lambda \lambda_{o f f} /\left(\lambda_{o f f}+1\right)$. We assume that the packet in a node is immediately sent to a "newly-born" node which has a

\footnotetext{
${ }^{1} \mathrm{~A}$ scaling factor can be applied to match the figures of a real deployment.
}

positive projection on the direction towards the $\operatorname{sink}^{2}$. We denote $\Delta_{n}=\left|X_{n+1}-X_{n}\right|$ where $X_{n}$ and $X_{n+1}$ are two successive node locations on the path of the packet sent towards the sink $\mathcal{S}$. We denote $S_{n}=T_{n+1}-T_{n}$ and $\theta_{n}$ is the angle between $\overline{X_{n} \mathcal{S}}$ and $\overrightarrow{X_{n} X_{n+1}}$.

The mean value of $\Delta_{n}, \cos \left(\theta_{n}\right)$ and $S_{n}$ can be easily computed: $E\left(\Delta_{n}\right)=\int_{0}^{\mathcal{R}_{\text {com }}}\left(1-\frac{r^{2}}{\mathcal{R}^{2}}\right) d r=\frac{2 \mathcal{R}_{\text {com }}}{3}, E\left(\cos \left(\theta_{n}\right)\right)=$ $\frac{1}{\pi} \int_{-\frac{\pi}{2}}^{\frac{\pi}{2}} \cos (\theta) d \theta=\frac{2}{\pi}, E\left(S_{n}\right)=\frac{\lambda_{o f f}+1}{\frac{\pi}{2} \mathcal{R}_{c o m}^{2} \lambda \lambda_{o f f}}$.

Thus the packet propagation speed is equal to:

$$
\mathcal{V}=\frac{E\left(\Delta_{n}\right) E\left(\cos \left(\theta_{n}\right)\right.}{E\left(S_{n}\right)}=\frac{2 \lambda \lambda_{o f f} \mathcal{R}_{c o m}^{3}}{3\left(1+\lambda_{o f f}\right)} .
$$

If we denote by $\mathcal{D}$ the Euclidian distance between the source and the sink, we can compute the mean number of hops as:

$$
\mathcal{N}_{\text {hop }}=\frac{\mathcal{D}}{E(\Delta) E(\cos \theta)}=\frac{3 \pi \mathcal{D}}{4 \mathcal{R}_{\text {com }}}
$$

The end-to-end packet delay can be easily expressed ${ }^{3}$ by:

$$
T_{\text {tot }}=\frac{\mathcal{D}}{\mathcal{V}}+\mathcal{N}_{\text {hop }} \times\left(T_{p k}+T_{b c}\right)
$$

where $T_{p k}$ and $T_{b c}$ are packet and beacon transmission delays respectively.

It is also possible to roughly evaluate $P_{\text {path }}$. A packet is not blocked on its path towards the sink if, at each hop, it finds an active node within its communication range (i.e. $\mathcal{R}_{\text {com }}$ ) with a positive projection on the direction towards the sink, and within the maximum waiting delay (i.e $1 / \lambda_{o f f}$ ). This occurs with a probability of: $1-\exp \left(-\frac{\pi \lambda \mathcal{R}_{\text {com }}^{2}}{2\left(\lambda_{\text {off }}+1\right)}\right)$. Thus we have:

$$
P_{\text {path }}=\left(1-\exp \left(-\frac{\pi \lambda \mathcal{R}_{c o m}^{2}}{2\left(\lambda_{\text {off }}+1\right)}\right)\right)^{\frac{3 \pi \mathcal{D}}{4 \mathcal{R}_{c o m}}}
$$

\section{Performance Evaluation}

We obtain the simulation results by considering a packet propagation from the source node $\mathcal{O}$ to the sink $\mathcal{S}$. For the simulation results, we use $\lambda=4000$ and $\lambda_{o f f}=0.01$. We set the packet's maximum waiting delay at each hop to 100 time units, which is equal to the mean sleeping period (i.e. $1 / \lambda_{o f f}$ ). We set the packet and the beacon transmission delays $T_{p k}$ and $T_{b c}$ to, respectively, 0.7 and 0.1 time unit. We set the simulation duration to 10000 time units. We study each parameter over an average of 100 simulations. Moreover, the results are always presented with error bars corresponding to a confidence level of $95 \%$.

To obtain real figures we may multiply the distances by 1000 , thus the network area is $1 \mathrm{~km}^{2}$ and the average distance between a node and its closest neighbour is approximately $15 \mathrm{~m}$. We may also assume that the sensor nodes use the CC2420 chipset and the same beacon frame as in IEEE 802.15.4 [6]. The transmit bit rate is equal to $250 \mathrm{bps}$ and the size of the beacon is equal to 19 bytes. In our model, a beacon transmission delay $T_{b c}$ is equal to 0.1 time unit. Thus 1 time unit is equal to $6.1 \mathrm{~ms}$. It is worth noting that we cannot compare the opportunistic routing with proactive (e.g. OLSR) or reactive (e.g. AODV) due to the asynchronous random on/off activity of transceivers within the

\footnotetext{
${ }^{2}$ With the opportunistic with delay variant, the selected node decreases the remaining distance to the sink which is slightly different here.

${ }^{3} \mathrm{We}$ add the transmission delay which is not considered in the analytical model.
} 


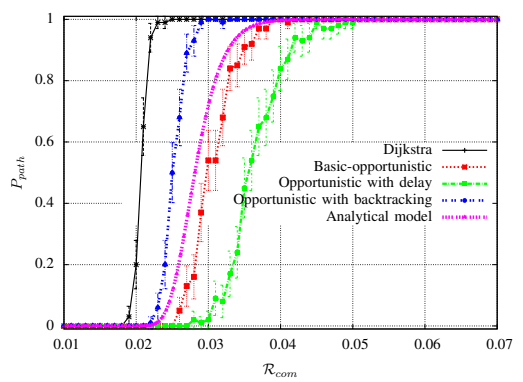

(a) $P_{\text {path }}$

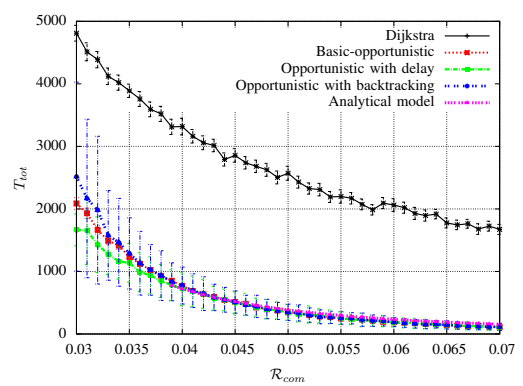

(b) $T_{\text {tot }}$

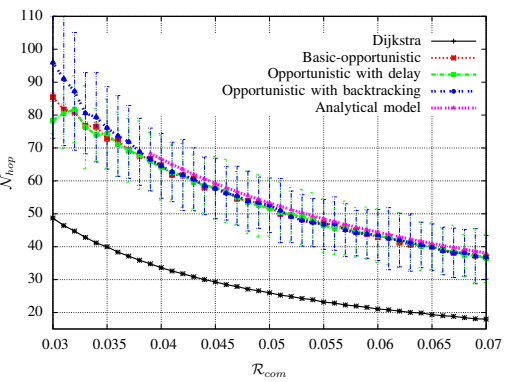

(c) $\mathcal{N}_{\text {hop }}$

Fig. 1. Performance comparison

network. In fact, routes change frequently and the route request does not exist when the route reply packet is sent.

In Fig. 1(a), we compare the probability of packet delivery, $P_{\text {path }}$, from the source node $\mathcal{O}$ to the sink $\mathcal{S}$ according to the sensors' transmission range, $\mathcal{R}_{\text {com }}$. We evaluate $P_{\text {path }}$ by simulation for the three opportunistic routing variants and Dijkstra's protocol. We observe that Dijkstra's packet delivery probability converges quickly $\left(\mathcal{R}_{c o m}=0.025\right)$ to 1 . If Dijkstra's protocol cannot convey a packet to the sink, a path does not exist in the physical network because Dijkstra's protocol computes all the possible routes. We remark that the opportunistic with backtracking protocol finds a path to the sink with a smaller communication range $\left(\mathcal{R}_{\text {com }}=0.030\right)$ than the two other variants. This is because with the opportunistic with backtracking protocol, the packet may be moved further back and is not blocked indefinitely in a node. Dijkstra's protocol only requires $\mathcal{R}_{\text {com }}>0.025$ thus the difference in the communication range required for the opportunistic with backtracking protocol and Dijkstra is very small. Moreover, based on extensive simulations, we notice that opportunistic with backtracking protocol moves back the packet by $5.89 \pm 0.86$ and $0.18 \pm 0.11$ hops when $\mathcal{R}_{\text {com }}$ is equal to 0.030 and 0.040 respectively. It is straightforward to see that opportunistic with backtracking protocol incurs a penny overhead. Besides, Fig. 1(a) shows that opportunistic with delay is the protocol that requires the largest communication range $\left(\mathcal{R}_{\text {com }}=0.051\right)$ to ensure $P_{\text {path }}=1$. Finally, we observe that all opportunistic variants can ensure packet delivery $\left(P_{\text {path }}=1\right)$ when the transmission range is large enough. Indeed, a delay does not affect a packet's progression to the sink.

In Fig. 1(b), we evaluate the packet's end-to-end delay, $T_{t o t}$, from the source node $\mathcal{O}$ to the sink $\mathcal{S}$. We remark that, with all the protocols, the end-to-end delay decreases when $\mathcal{R}_{\text {com }}$ increases. Moreover, we notice that all opportunistic routing variants notably outperform Dijkstra's protocol and the gain is approximately between 2 and 4 times. In fact, Dijkstra's algorithm builds a shortest path in terms of hops but not in terms of delay. Since sensors' transceivers use duty-cycles, a packet waits in each node for a long period, until the next node computed by Dijkstra's protocol wakes up. Moreover, we note that when $\mathcal{R}_{\text {com }}$ is small, opportunistic with delays is the fastest protocol. However, its packet delivery probability remains smaller than the two other variants. Opportunistic with backtracking routing is the protocol which exhibits the largest delivery delay which occurs because the packet can be moved further back from the sink. Hence, the path generated is longer than the other opportunistic variants (as shown in Fig. 1(c)). However, we observe that all the opportunistic variants have the same packet end-to-end delay when $\mathcal{R}_{\text {com }}$ is large.

In Fig. 1(c), we compare the average length of the paths, $\mathcal{N}_{\text {hop }}$, obtained by the simulations. We notice that opportunistic routing protocols never more than double the length of the path between the source node $\mathcal{O}$ and the sink $\mathcal{S}$ compared to Dijkstra's protocol. Nevertheless, as already pointed out and shown in Fig. 1(b), the end-to-end delay is notably decreased (i.e. the gain is roughly between 2 and 4 times). Moreover, we observe that with small values of $\mathcal{R}_{\text {com }}$, opportunistic with backtracking has the longest path which can be explained by the fact that the packet may move further back from the sink. In addition, we observe that when $\mathcal{R}_{\text {com }}$ increases, all the opportunistic routing variants have the same path length.

\section{CONCLUSION}

In this paper, we have studied the performance of crosslayer geographic opportunistic protocol based on the RI-MAC protocol. The scheme is designed to operate in low-duty cycle WSNs. The routing uses an opportunistic approach and we have considered three variants denoted by i) basic-opportunistic, ii) opportunistic with delay and iii) opportunistic with backtracking. The performance of the opportunistic cross-layer scheme has been studied with simulations and analytical model to calculate the i) probability of packet delivery, ii) end-to-end packet forwarding delay and iii) average path-length. The results obtained show that opportunistic routing is between two and four times more efficient than the shortest path algorithm in terms of end-to-end packet forwarding delay and it is never more than doubles the length of the path. REFERENCES

[1] F. Baccelli, B. Blaszczyszyn, and P. M. Zeimpekis, "Time-space opportunistic routing with an optimized relay self selection technique in wireless ad hoc networks," Computer Journal, June 2009.

[2] P. Jacquet, B. Mans, P. Muhlethaler, and G. Rodolakis, "Opportunistic routing in wireless ad hoc networks: upper bounds for the packet propagation speed," IEEE Journal on Selected Areas in Communications, vol. 27, pp. 1192-1202, 2009.

[3] Y. Sun, O. Gurewitz, and D. B. Johnson, "RI-MAC: A receiver initiated asynchronous duty cycle MAC protocol for dynamic traffic loads in wireless sensor networks," SenSys: ACM Conference on Embedded Networked Sensor Systems, pp. 1-14, 2008.

[4] V. Zeimpekis, G. M. Giaglis, and G. Lekakos, "A taxonomy of indoor and outdoor positioning techniques for mobile location services," ACM - SIGecom Exch., vol. 3, pp. 19-27, 2003.

[5] B. Błaszczyszyn and P. Mühlethaler, "Stochastic analysis of non-slotted Aloha in wireless ad-hoc networks," in Proc. of IEEE INFOCOM, San Diego, CA, 2010.

[6] "IEEE standard, part 15.4: Wireless medium access control (mac) and physical layer (phy) specifications for low-rate wireless personal area networks (lr-wpans)," IEEE Computer Society, 2003. 